\title{
Indépendance de Bank Al-Maghrib : Un essai d'évaluation en utilisant la méthodologie de Cukierman, Webb et Neyapti
}

\author{
Moulaydriss SAIKAK ${ }^{1}$ and Radouane RAOUF ${ }^{2}$ \\ ${ }^{1,2}$ Economic department, University Mohamed V, Rabat, Morocco
}

Résumé : La majorité des banques centrales ont révisés leurs statuts afin d'obtenir plus d'indépendance en optant pour la stabilité des prix en tant qu'objectif prioritaire. Bank Al-Maghrib, BAM, ne s'est pas épargnée par cette vague de réforme institutionnelle et s'est dotée d'un nouveau statut qui lui confère plus d'indépendance en 2006. L'analyse du degré de son indépendance en utilisant la méthodologie proposée par Cukierman, Webb et Neyapti (1992), qui s'avère un indice approprié vu sa double vocation en termes de mesure légale et réelle, a montré qu'effectivement BAM est une banque relativement indépendante de point de vue de jure et de facto.

Mots-clés : Banque centrale, indépendance, Mesure de Jure, Mesure de facto.

\section{INTRODUCTION:}

La nouvelle synthèse macroéconomique établit grâce aux travaux de Lucas, de Kydland et Prescott (1977) et Barro et Gordon (1983), a consacré l'indépendance de la banque centrale. Dans cet élan, plusieurs banques centrales ont amendé leurs statuts pour en obtenir plus d'indépendance et ont opté pour un objectif prioritaire de stabilité des prix, et par conséquent la maîtrise de l'inflation.

Tester statistiquement la relation qui existe entre l'indépendance de la banque centrale et l'inflation revient à rendre le degré d'indépendance quantifiable. De ce fait, il est légitime de distinguer le statut juridique et la responsabilité politique. L'indépendance de jure et l'indépendance de facto, et par conséquent la relativité de l'indépendance de la banque centrale.

L'adoption d'une règle pour conduire une politique monétaire constitue l'ossature et le cadre de référence des actions entreprises par l'autorité monétaire et un modèle pour les anticipations des agents 
privés. Le degré d'indépendance dépend du cadre institutionnel des règles et de l'interprétation qu'en font les différents centres de l'exécutif de la banque centrale, dépend de la doctrine monétaire et de la culture démocratique de chaque pays.

La mesure légale ou de jure, c'est-à-dire le degré d'indépendance que le législateur, à travers les textes de loi, attribue à la banque centrale s'avère très réaliste. Depuis les travaux de Bade et Parkin (1980) qui sont les premiers économistes ayant tenté de quantifier le degré de l'indépendance de la banque centrale à travers la construction d'un indicateur en se basant que sur les textes de loi qui forment leurs statuts. Tous les auteurs en la matière ont basé leurs travaux sur la même méthodologie. Le problème que rencontrent les spécialistes est que les lois des banques centrales diffèrent par leur objet, leur portée et leur degré de détails. Les différents statuts des banques centrales qui sont aussi divers que le sont les traditions législatives des nations rendent l'exercice plus difficile (Aglietta (1992)). Il est donc difficile de classer les statuts des banques centrales en fonction de leur degré d'indépendance légale, ce qui exige inévitablement un jugement subjectif.

La recherche d'une relation entre les statuts des banques centrales et les résultats de la politique monétaire a pour objectif d'instaurer un cadre de politique monétaire qui offre les meilleures performances macroéconomiques.

Notre étude a pour objectif l'évaluation du degré d'indépendance de BAM en utilisant la mesure de jure et la mesure de facto proposées par Cukierman, Webb et Neyapti, CWN. Elle est divisée en deux parties combinant la théorie et la pratique. D'abord, un aperçu sur la méthodologie de construction de l'indice composite de CWN est évoqué dans la première partie. puis un essai d'évaluation du degré de l'indépendance de BAM fera l'objet de la deuxième partie.

\section{APERÇU HISTORIQUE DE BANK AL-MAGHRIB}

Comme tous autres pays, le Maroc s'est vu doté d'un organisme dont la mission est la conduite de la politique monétaire. C'est en 1906, et exactement le 07 Avril de la même année, que l'institution monétaire a vu le jour avec la dénomination de « la banque d'État du Maroc ». Cette banque d'émission internationale créée par l'acte d'Algésiras de 1906 dans le contexte des rivalités impérialistes de notre première mondialisation. Le discours prononcé par le roi Mohammed V le 2 juillet 1959 marque la création d'une autorité monétaire «La souveraineté de toute nation se manifeste par des attributs, notamment la liberté d'action dans la conduite de la politique financière et monétaire et l'orientation de son économie conformément à ses intérêts, et par la possession d'une monnaie nationale émise par une banque nationale et non une monnaie satellite émise par un institut étranger. [...] Notre pays se trouve ainsi doté d'une institution nationale dirigée par des nationaux ». Le 30 juin 1959, le Maroc instaure sa propre banque centrale dont la dénomination est la banque du Maroc en remplacement à la banque d'État du Maroc et ce n'était qu'en 1987, 03 mars, que le Maroc a adopté la dénomination « Bank Al-Maghrib ». Le 17 octobre 
1959 est institué le dirham, nouvelle unité monétaire du Maroc en remplacement du franc marocain introduit en 1921.

Suite l'effondrement, au cours des années soixante-dix, du système économique international, des programmes d'ajustement structurel ont été imposé durant les années quatre-vingt et ont obligé toutes les économies nationales à se transformer pour s'ajuster au nouvel état de l'économie mondiale.

Afin de renforcer la stabilité financière et à moderniser le système bancaire en vue d'accompagner l'intégration de l'économie marocaine dans l'environnement international, BAM s'est vue dotée d'un nouveau statut qui a fait l'objet de la loi n 76-03 entrée en vigueur le 20 février 2006. Ce nouveau statut a redéfini et élargi les missions fondamentales en matière de politique monétaire.

\section{MESURE DE JURE DE L'INDEPENDANCE DE LA BANQUE CENTRALE}

Cukierman, Webb et Neyapti, comme leurs prédécesseurs, ont développé deux (02) indices d'indépendance de la banque centrale. En effet, pour un échantillon de 72 pays (21 pays industrialisés et 51 pays en voie de développement) un indice légal, LVAW (Annexe 1), et l'indice de rotation des gouverneurs des banques centrales (Turnover : TOR) sont construites.

\section{MESURE DE FACTO DE L'INDEPENDANCE DE LA BANQUE CENTRALE}

\section{A. INDICE DE ROTATION DU GOUVERNEUR DE CUKIERMAN (1992)}

Cukierman (1992) propose un indicateur de mesure de facto de l'indépendance de la banque centrale, l'indice de rotation du gouverneur (TOR). Pour un échantillon de 58 économies dont 19 des économies avancées et 39 des pays en voie de développement pour une période allant de 1950 à 1989, l'auteur s'est servi d'une formule assez élémentaire pour le taux de rotation du gouverneur, en grande partie à cause de l'absence d'indicateurs qui permettent de mesurer dans quelle mesure le degré d'autonomie réelle des BC s'écarte de l'autonomie stylisée rencontrée dans les statuts juridiques des différents pays. Un taux de rotation plus rapide du gouverneur de la banque centrale indique un niveau inférieur d'autonomie.

Le taux de rotation du gouverneur (TOR) est défini comme le nombre annuel moyen de roulement des gouverneurs de la banque centrale. Les TOR vont d'un minimum de 0,03 (un mandat moyen de 33 ans) pour l'Irlande à un maximum de 0,93 (un mandat moyen de 18 mois) pour l'Argentine.

Pour les pays de l'OCDE, la fourchette est de 0,03 pour l'Islande à 0,20 pour le Japon et l'Espagne, plus de la moitié des pays moins développés dépasse ce maximum, la fourchette variante de 0,13 pour la Malaisie à 0,93 pour l'Argentine. Un taux de rotation élevé semble indiquer une faible autonomie lorsque le mandat effectif du gouverneur de la banque centrale est plus court que le mandat effectif de l'exécutif. 


\section{B. INDICE DE LA VULNERABILITE POLITIQUE DU GOUVERNEUR}

Cukierman et Webb (1995) ont proposé un autre indice de mesure du degré réel de l'indépendance de la banque centrale, l'indice de vulnérabilité politique du gouverneur. Pour une période allant de 1950 à 1989 et un échantillon hétérogène composé de 20 pays de l'OCDE et 47 pays moins développés, les deux auteurs ont subdivisé la période en deux sous période, avant Bretton-Woods et après c'est-à-dire de 1950 à 1971 et 1971 à 1989.

La fréquence de rotation de la direction de la banque centrale est calculée sur plusieurs intervalles de temps tout en s'éloignant d'une transition politique. après avoir prouvé empiriquement que les changements dans la direction d'une banque centrale, qui peuvent être définis comme purement politiques, sont ceux qui se produisent dans les six mois suivant le changement du pouvoir exécutif, et après avoir calculé la fréquence annuelle des changements politiques dans chaque pays, Cukierman et Webb définissent leur indicateur de vulnérabilité de la BC à l'instabilité politique comme le pourcentage de transition politiques dans un pays qui ont été immédiatement suivies par le remplacement du gouverneur de la BC :

$\mathrm{V}(\mathrm{i})=$ Nombre de remplaçants du gouverneur de la $\mathrm{BC}$ dans les i mois qui suivent une transition politique Avec $1<\mathrm{i}<6$

\section{EVALUATION DU DEGRE D'INDEPENDANCE DE BAM}

\section{A. MESURE DE JURE DE L'INDEPENDANCE DE BAM}

Comme déjà indiqué ci-dessus, l'indice légal est apprécié par rapport aux textes de lois ${ }^{1}$ que le législateur confère à BAM. L'indice de $\mathrm{CWN}$ comporte quatre caractéristiques :

$>$ Indépendance des dirigeants, formulation de la politique monétaire, objectifs attribués à la banque centrale et les limitations des crédits au gouvernement.

- L'analyse de l'indépendance des dirigeants de la banque centrale mène aux résultats suivants : la durée du mandat est de six ans renouvelable une seule fois en se référant à l'article 30 du statut de BAM, donc on lui attribue le score de 0,75 .

- La procédure de nomination du gouverneur est prévue par l'article 49 de la constitution Marocaine. Donc c'est le pouvoir législatif qui se charge de la nomination, un score de 0,50 lui est attribué.

\footnotetext{
${ }^{1}$ Le nouveau statut de BAM Objet de la loi n 76-03 entrée en vigueur le 20 février 2006
} 
- Conditions de limogeage du gouverneur ou des membres du conseil ne sont pas prévues par les statuts. Un score de 1 est donné.

- Le gouverneur ou les membres du conseil ne peuvent exercer d'autres fonctions en vertu de l'article 38 deuxième alinéa. Le score de 1 est attribué.

- En vertu de l'article 6 des statuts de BAM, ce dernier définit et conduit en toute transparence, la politique monétaire, on lui attribue le score de 1.

- En relation avec le poids de la banque centrale dans la résolution des conflits avec le gouvernement, la banque a le dernier mot concernant ses objectifs tels que définis par la loi. Un score de 1 lui est attribué.

- BAM n'intervient pas dans le processus budgétaire, le score de 0 lui est dédié.

- L'objectif principal attribué à la banque centrale est de maintenir la stabilité des prix. cependant, on attribue le score de 1 .

$>$ Les limitations des crédits au gouvernement, elles sont caractérisées par :

- Les avances monétaires au gouvernement: l'article 69 des statuts de BAM prévoit que des concours financiers sous forme de facilités de caisse seront accordé et sont limitées à $5 \%$ des recettes fiscales réalisées au cours de l'année budgétaire écoulée. Le score de 0,67 est attribué. Les avances contre titres ne sont pas permises. En effet, en vertu du premier alinéa de l'article 69, BAM ne peut se porter garante d'engagements contractés par l'État, acquérir directement des titres de créances. Le score de 1 est attribué.

- Les conditions d'emprunt (maturité, intérêt, montant) sont prévues dans les statuts de BAM en vertu de l'article 69. On attribue un score de 0,67.

- Les emprunteurs potentiels auprès de BAM sont tant le secteur privé que public. Un score de 0 est attribué.

- Les limites de concours de BAM au gouvernement définies comme une facilité de caisse à hauteur de $5 \%$ des recettes fiscales. En revanche, le score de 0,33 est attribué.

- Maturité des prêts, en vertu du deuxième alinéa de l'article 69 , ne peut excéder 120 jours soient quatre mois. Le score de 1 est dédié.

- Les taux d'intérêt sur les avances devraient être rémunérés au taux de base de refinancement des banques auprès de BAM. Le score 0,75 est affecté.

- BAM est autorisée à acheter ou vendre les titres publics sur le marché primaire en vertu de l'article 66 des statuts de BAM. Le score 1 est attribué.

La réforme de 2006 des statuts de la BAM lui confère plus d'indépendance dans la conduite de la politique monétaire de point de vue légal. En effet, suivant aux statuts de BAM de 1993, CWN ont trouvé un score d'indice légal de 0,14 tandis que suivant aux statuts de 2006, on a trouvé un indice de 0,757 qui s'éloigne de 0 et s'approche de 1 . Il reste à évaluer le degré d'indépendance à travers l'évaluation de la vraie conduite 
de la politique monétaire. Pour cela, on utilise le taux de rotation du gouverneur de la banque centrale et

l'indice de vulnérabilité politique du gouverneur.

La table 1 récapitule le poids et le score de chaque composante de l'indice CWN.

Table 1. Construction de l'indice légal de $\mathrm{CWN}$ relatif à BAM

\begin{tabular}{l} 
Caractéristiques \\
\hline 1- Indépendance des dirigeants : \\
\hline a- $\quad$ Durée du mandat : \\
$\mathbf{6}$ à 8 ans \\
b- Procédure de nomination du gouverneur : \\
Pouvoir législatif ; \\
c- Conditions de limogeage du gouverneur ou des membres du \\
conseil : \\
Non-prévu par la loi ; \\
d- Le gouverneur ou les membres du conseil peuvent-ils exercer \\
d'autres fonctions?
\end{tabular}

Non ;

\begin{tabular}{lll} 
Poids & Score & Poids*score \\
\hline 0,20 & & 0,1625 \\
\hline 0,05 & 0,75 & 0,0375 \\
0,05 & & \\
0,05 & 0,50 & 0,025 \\
0,05 & & \\
& 1,00 & 0,05 \\
& & \\
\hline 0,15 & 1,00 & 0,05 \\
\hline 0,05 & 1,00 & 0,1 \\
0,05 & 1,00 & 0,05 \\
& & 0,05 \\
\hline
\end{tabular}

par la loi ;

c- Participation de la $\mathrm{BC}$ au processus budgétaire :

La BC n'intervient pas.

0,05

a- Qui formule la politique monétaire?

\begin{tabular}{|c|c|c|c|}
\hline 3- Objectifs attribués à la banque centrale & $\mathbf{0 , 1 5}$ & & $\mathbf{0 , 1 5}$ \\
\hline $\begin{array}{l}\text { La stabilité des prix mentionnée comme objectif unique ou } \\
\text { prioritaire, la BC ayant le dernier mot en cas de conflit d'objectifs } \\
\text { (autre objectif : plein emploi, etc. }\end{array}$ & 0,15 & $\mathbf{1 , 0 0}$ & 0,15 \\
\hline 4- Limitations des crédits au gouvernement : & $\mathbf{0 , 5 0}$ & & 0,3445 \\
\hline $\begin{array}{l}\mathrm{a}-\quad \text { Les avances monétaires au gouvernement : } \\
\text { Avances permises mais dans des limites strictes (maximum des avances = } \\
\mathbf{5 \%} \text { des revenus du gouvernement) }\end{array}$ & 0,15 & 0,67 & 0,1005 \\
\hline b- Avances contre titres: & 0,10 & & \\
\hline $\begin{array}{l}\text { Pas permis } \\
\text { c- Conditions d'emnrunt (maturité intérêt montant). }\end{array}$ & & 1,00 & $\mathbf{0 , 1 0}$ \\
\hline $\begin{array}{l}\text { c- Conditions d'emprunt (maturité, intérêt, montant) : } \\
\text { Spécifiées par les statuts de la BC }\end{array}$ & 0,10 & & \\
\hline d- Emprunteurs potentiels auprès de la $\mathrm{BC}$ : & & 0,67 & 0,067 \\
\hline $\begin{array}{l}\text { Tant bien le secteur public que privé } \\
\text { e- Les limites de concours de la BC au gouvernement définies comme }\end{array}$ & 0,05 & $\mathbf{0 , 0 0}$ & $\mathbf{0 , 0 0}$ \\
\hline Fractions du revenu du gouvernement & 0,025 & & \\
\hline $\begin{array}{l}\mathrm{f}-\quad \text { Maturité des prêts : } \\
<\mathbf{6} \text { mois }\end{array}$ & & $\mathbf{0 , 3 3}$ & 0,00825 \\
\hline $\begin{array}{l}\text { g- Les taux d'intérêts sur les avances devraient être : } \\
=\text { taux de marché }\end{array}$ & 0,025 & 1,00 & 0,025 \\
\hline $\begin{array}{l}\text { h- La BC est-elle autorisée à acheter ou vendre les titres publics sur le marché } \\
\text { primaire? }\end{array}$ & 0,025 & $\mathbf{0 , 7 5}$ & $\mathbf{0 , 0 1 8 7 5}$ \\
\hline Oui & 0,025 & 1,00 & 0,025 \\
\hline Score total & & & 0,757 \\
\hline
\end{tabular}

Source: établi par les auteurs. 


\section{B. MESURE DE FACTO D'INDEPENDANCE DE BAM}

\section{TAUX DE ROTATION DU GOUVERNEUR}

Les deux mesures de la vraie conduite de la Banque Centrale utilisées par Cukierman (1992) sont le taux de rotation du gouverneur et sa vulnérabilité politique.

Pour le cas du Maroc, les statuts de BAM stipulent que le gouverneur de la banque centrale est nommé pour 6 ans, donc le taux moyen de rotation du gouverneur est de 0.16 (un changement par 6 ans).

En se référant à la table 2, le taux de rotation du gouverneur de la banque centrale du Maroc est de 0.13 soit un changement chaque sept ans et demi. On constate que la tenure du gouverneur de la banque centrale dans son poste présente une nette volatilité. En effet, Driss SLAOUI, M'Hamed Zghari n'ont pas terminé leurs mandats (ils sont respectivement restés dans leurs postes 4 ans et 3 ans).

Du tableau 7, le Taux moyen de rotation est :

Nombre de changements total / durée de la période $=6 / 45=0,13$

À noter que le taux de rotation relatif au dernier gouverneur Mr, Abdellatif JOUAHRI n'est pas inclus dans les calculs puisqu'il est encore dans son poste.

Table 2. Taux de rotation du gouverneur de BAM

\begin{tabular}{|c|c|c|}
\hline Gouverneur & Taux de rotation du gouverneur & \\
\hline $\begin{array}{l}\text { M.M'hamed Zghari } \\
\text { 1959-1964 }\end{array}$ & 0,16 & \multirow{11}{*}{$\begin{array}{l}\text { Nombre de changements total } \\
\text { / durée de la période : }\end{array}$} \\
\hline $\begin{array}{l}\text { M. Driss SLAOUI } \\
1964-1967\end{array}$ & 0,25 & \\
\hline $\begin{array}{l}\text { M.M'hamed Zghari } \\
\text { 1967-1969 }\end{array}$ & 0,33 & \\
\hline $\begin{array}{l}\text { Son Altesse le prince Moulay } \\
\text { Hassan BEN EL MEHDI } \\
\text { 1969-1974 }\end{array}$ & 0,16 & \\
\hline $\begin{array}{l}\text { Son Altesse le prince Moulay } \\
\text { Hassan BEN EL MEHDI } \\
\text { 1975-1981 }\end{array}$ & 0,16 & \\
\hline $\begin{array}{l}\text { Son Altesse le prince Moulay } \\
\text { Hassan BEN EL MEHDI } \\
\text { 1982-1984 }\end{array}$ & 0,33 & \\
\hline $\begin{array}{l}\text { M. Ahmed BENANI } \\
\text { 1985-1989 }\end{array}$ & 0,2 & \\
\hline $\begin{array}{l}\text { M. Mohamed SEQAT } \\
\text { 1989-1994 }\end{array}$ & 0,16 & \\
\hline $\begin{array}{l}\text { M. Mohamed SEQAT } \\
\text { 1995-2000 }\end{array}$ & 0,16 & \\
\hline $\begin{array}{l}\text { M. Mohamed SEQAT } \\
2001-2003\end{array}$ & 0,33 & \\
\hline $\begin{array}{l}\text { M. Abdellatif JOUAHRI } \\
2003 \text { à nos jours }\end{array}$ & Est encore gouverneur & \\
\hline
\end{tabular}




\section{II- LA VULNERABILITE POLITIQUE DU GOUVERNEUR}

Trois cas de vulnérabilité politique du gouverneur de la banque centrale du Maroc peuvent être distingués (Tableau 3). En effet, la nomination de Abdellah IBRAHIM le 24 décembre 1958 est suivi par l'arrivée de M'Hamed Zghari le 1er juillet 1959 dans le poste de gouverneur de la banque centrale. Le deuxième cas est relatif au Premier ministre El Hadj Ahmed BAHNINI arrivé à sa fonction le 3 novembre 1963. Un changement du gouverneur s'est fait par la nomination de Driss SLAOUI en 1964. Le troisième cas se concrétise par la nomination de M'Hamed Zghari en 1967 suite à l'arrivée du gouvernement de Mohamed BENHIMA le 6 juillet 1967. Ainsi, le ratio de vulnérabilité politique prend la valeur $(3 / 15)=0,2$ puisqu'il y avait 15 changements des premiers ministres et seulement 3 changements des gouverneurs de la banque centrale dans un délai ne dépassant pas 6 mois.

Table 3. Vulnérabilité politique du gouverneur de BAM

\begin{tabular}{|c|c|}
\hline Nomination des gouverneurs de la Banque Centrale & Les premiers ministres Marocains \\
\hline & $\begin{array}{l}10 \text { octobre } 2013 \\
\text { Abdelilah BENKIRANE } \\
9 \text { novembre } 2011 \\
\text { Abdelilah BENKIRANE } \\
19 \text { Septembre } 2007 \\
\text { Abbas EL FASSI } \\
7 \text { Novembre } 2002 \\
\text { Driss JETTOU } \\
8 \text { juin } 2004 \\
\text { Driss JETTOU } \\
14 \text { mars } 1998 \\
\text { Abderrahmane YOUSSOUFI }\end{array}$ \\
\hline > M'Hamed Zghari (1er juillet 1959 au 20 août 1964) & 6 septembre 2000 \\
\hline$>\quad$ M. Driss SLAOUI (1964-1967) & $\begin{array}{l}\text { Abderrahmane YOUSSOUFI } \\
27 \text { février } 1995 \\
\text { Abdellatif FILALI }\end{array}$ \\
\hline$>\quad$ M'Hamed Zghari (1967) & $\begin{array}{l}7 \text { juin } 1994 \\
\text { Abdellatif FILALI } \\
30 \text { septembre } 1986\end{array}$ \\
\hline $\begin{aligned} & \text { Le prince Moulay Hassan Ben El Mehdi } \\
& \text { (11 février } 1969 \text { au 1er novembre 1984) }\end{aligned}$ & $\begin{array}{l}\text { Azzedine LARAKI } \\
11 \text { aout } 1992 \\
\text { Mohamed Karim LAMRANI } \\
19 \text { novembre } 1983\end{array}$ \\
\hline$>\quad$ Ahmed Bennani (1er avril 1985, septembre 1989) & $\begin{array}{l}\text { Mohamed Karim LAMRANI } \\
12 \text { avril } 1972 \\
\text { Mohamed Karim LAMRANI } \\
6 \text { Aout } 1971\end{array}$ \\
\hline$>\quad$ Mohammed Seqat (septembre 1989 à 2003). & $\begin{array}{l}\text { Mohamed Karim LAMRANI } \\
5 \text { Novembre } 1981 \\
\text { Maati BOUABID } \\
27 \text { Mars } 1979 \\
\text { Maati BOUABID }\end{array}$ \\
\hline$>\quad$ Abdellatif JOUAHRI (depuis mars 2003) & $\begin{array}{l}10 \text { Octobre } 1977 \\
\text { Ahmed OSMAN } \\
20 \text { November } 1972 \\
\text { Ahmed OSMAN } \\
7 \text { October } 1969 \\
\text { Ahmed LARAKI } \\
\text { 6 Juillet } 1967\end{array}$ \\
\hline
\end{tabular}




Mohamed BENHIMA
3 November 1963
EI Hadj Ahmed BAHNINI
24 décembre 1958
Abdellah IBRAHIM
12 Mai 1958
El Hadj Ahmed BALAFREJ
28 Octobre 1956
M'barek Lahbil EL BEKKAI
7 Décembre 1955
M'barek Lahbil EL BEKKAI

Source : établi par les auteurs à partir des données de BAM et le site web du chef du gouvernement

\section{CONCLUSION}

L'indépendance de la banque centrale se manifeste par la non-interférence des décisions des décideurs politiques et les autorités monétaires.

Pour pouvoir tester la relation entre l'indépendance de la banque centrale, plusieurs auteurs ont proposé des mesures qu'on peut les subdiviser en deux. Des mesures de jure et des mesures de facto. Ces mêmes auteurs et d'autres ont testé la relation entre l'indépendance de la banque centrale et la performance macroéconomique. Ils ont trouvé des résultats divergents tandis qu'on théorie l'indépendance de la banque centrale engendre une inflation faible. En théorie, l'indépendance de la banque centrale est gage de crédibilité de la politique monétaire mais afin de conforter cette crédibilité, la transparence de l'autorité monétaire s'avère de grande importance.

Le degré d'indépendance de BAM a été apprécié au moyen de trois indices proposés par Cukierman, Webb et Neyapti (1992). Il s'est avéré que, bien que, récemment BAM se soit assurée comme objectif principal la stabilité des prix, et commence à être plus transparente. En effet, en utilisant trois indices mesurant le degré d'indépendance de la banque centrale à savoir; l'indice légal, l'indice de rotation du gouverneur et l'indice de vulnérabilité politique du gouverneur, il semble que la banque centrale du Maroc a connu une amélioration de son degré d'indépendance.

En théorie, l'indépendance de la banque centrale est synonyme de l'efficacité de la politique monétaire. Ce constat nous amène à évaluer l'efficacité de la politique monétaire dans le cadre de l'indépendance de l'autorité monétaire. 


\section{Annexes}

Annexe 1 : Construction de l'indice légal de CWN.

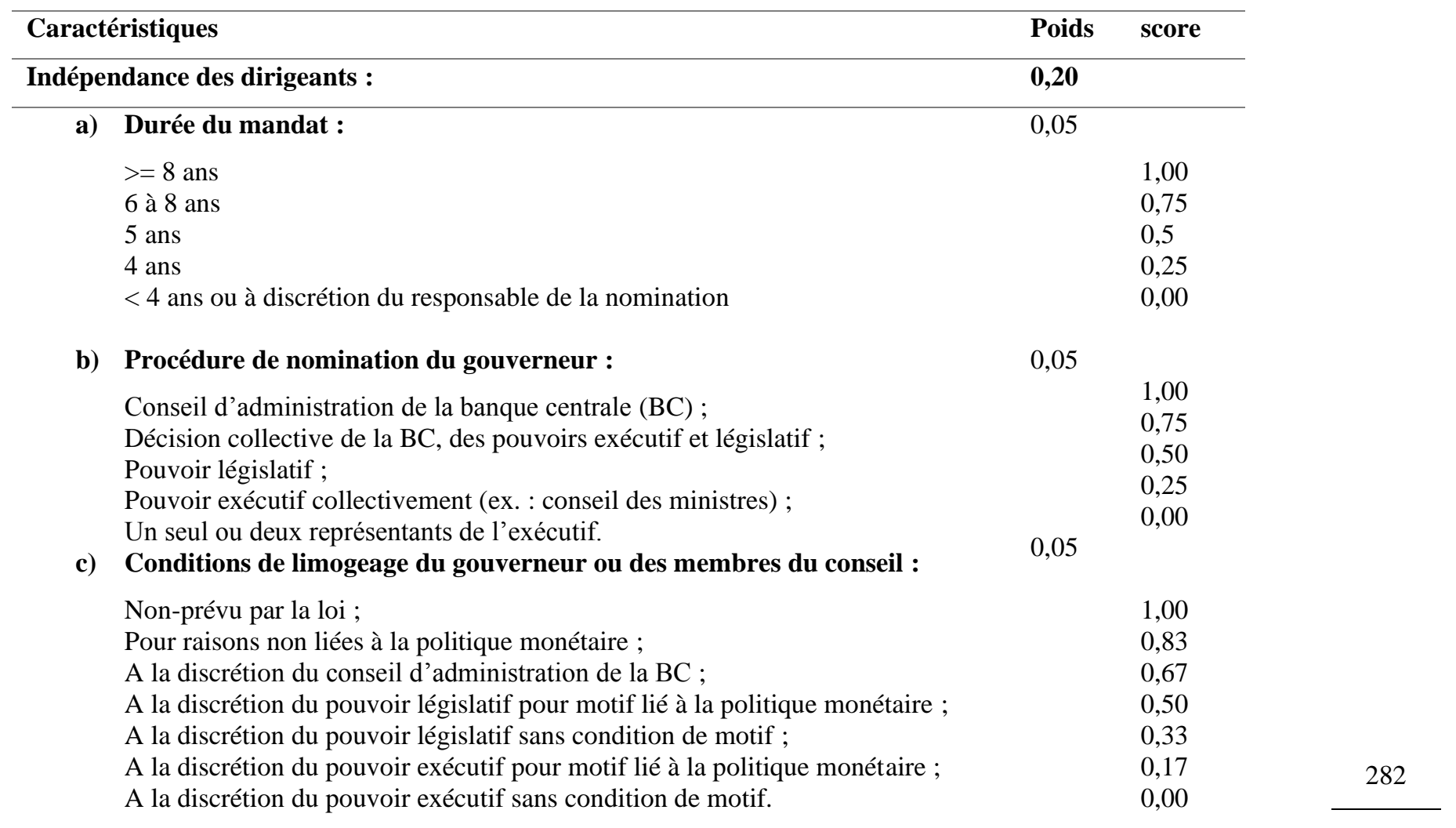

d) Le gouverneur ou les membres du conseil peuvent-ils exercer d'autres fonctions?

Non ;

Seulement avec préalable autorisation du pouvoir exécutif ; $\quad 0,50$ Aucune réglementation en la matière.

0,00

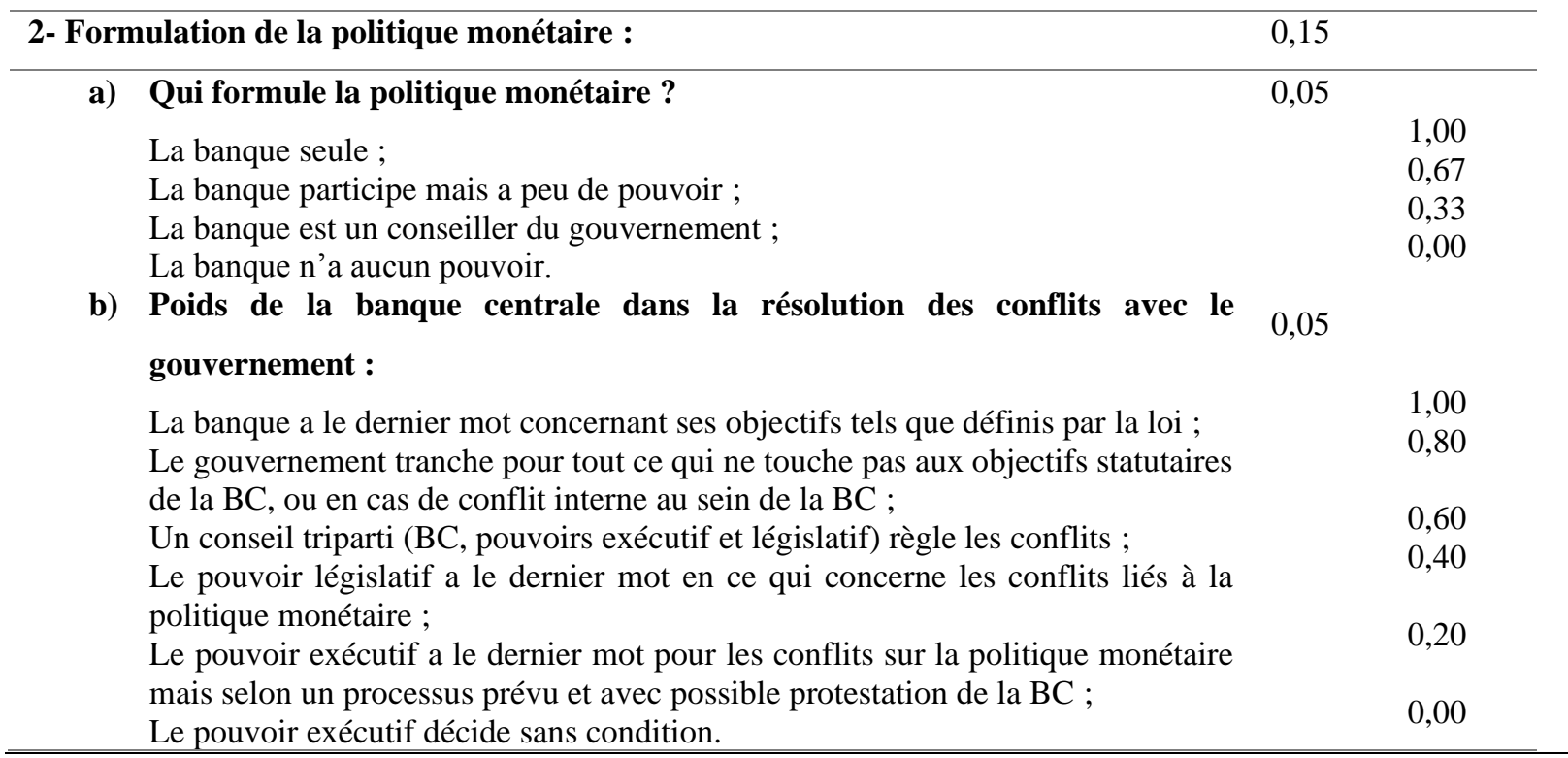


c) Participation de la $\mathrm{BC}$ au processus budgétaire :

La BC joue un rôle actif

0,05

La BC n'intervient pas.

1,00

0,00

3- Objectifs attribués à la banque centrale : $\quad 0,15$

a) La stabilité des prix mentionnée comme objectif unique ou prioritaire, la BC ayant le dernier mot en cas de conflit d'objectifs (autre objectif : plein emploi, etc.)

1,00

b) La stabilité des prix est l'unique objectif ;

0,80

0,60 telle la solidité du système bancaire ;

d) La stabilité de la valeur de la monnaie est associée à d'autres objectifs qui lui sont $\quad 0,40$ incompatibles ;

$\begin{array}{ll}\text { e) Aucun objectif spécifié ; } & 0,20\end{array}$

f) Objectifs autres que la stabilité des prix.

4- Limitations des crédits au gouvernement :

a- Les avances monétaires au gouvernement :

Aucune avance n'est autorisée

0,00

Avances permises mais dans des limites strictes (ex : maximum des avances $=$ $15 \%$ des revenus du gouvernement)

Avances permises avec des limites plutôt lâches (ex : maximum des avances = au-delà de $15 \%$ des revenus du gouvernement)

Pas de limites légales

b- Avances contre titres :

Pas permis

Permis mais dans des limites strictes (ex : maximum des avances $=15 \%$ des revenus du gouvernement)

Permis avec des limites plutôt lâches (ex : maximum des avances $=$ au-delà de

$15 \%$ des revenus du gouvernement)

Pas de limites légales

$\mathbf{0 , 5 0}$

0,15

1,00

0,67

Conditions d'emprunt (maturité, intérêt, montant) :

Fixées unilatéralement par la BC

Spécifiées par les statuts de la BC

Négociées par le gouvernement avec la BC

Imposées par le gouvernement

d- Emprunteurs potentiels auprès de la $\mathrm{BC}$ :

Le gouvernement central uniquement

Tous les niveaux du gouvernement

Le secteur public (Tous les niveaux du gouvernement plus les entreprises publiques)

Tant bien le secteur public que privé

0,33

0,00

$\mathbf{0 , 1 0}$

1,00

0,67

0,33

0,00

$\mathbf{0 , 1 0}$

1,00

0,67

0,33

0,00

0,05

1,00

0,67

0,33

0,00

e- Les limites de concours de la $\mathrm{BC}$ au gouvernement définies comme :

Montants d'argent

Fractions du capital ou du passif de la BC ;

Fractions du revenu du gouvernement

Fractions des dépenses du gouvernement

f- Maturité des prêts :

1,00

0,67

0,33

0,00

$<6$ mois

$<1$ an

1,00

0,67

$>1$ an

0,33

0,00

Non-définie dans la loi.

0,025

g- Les taux d'intérêts sur les avances devraient être :

$>$ au taux minima

0,50

$=$ taux de marché

0,25 
Aucune mention de taux d'intérêt

0,00

Pas d'intérêt perçu sur les avances au gouvernement

h- La BC est-elle autorisée à acheter ou vendre les titres publics sur le marché $\quad 0,025$ primaire?

Oui

1,00

Non

\section{BIBLIOGRAPHIE}

[1] Aglietta, Michel. «L'indépendance des banques centrales / Leçons pour la banque centrale européenne.» Revue d'économie financière, No. 22 (1992): 37-56.

[2] «L'indépendance des banques centrales : Leçons pour la banque centrale européenne.» Revue d'économie financière (1992): 37-56.

[3] Aguir, Abdelkader. «L'indépendance réelle de la banque centrale et le biais inflationniste : une validation empirique.» HAL Id: halshs-00800059 (2013).

[4] Alex Cukierman, Steven B. Webb and Biilin Neyapti. «Measuring the independence of central banks and its effect on policy outcomes.» The world bank (1992).

[5] Alex, Cukierman. «Central Bank Strategy, Credibility, and Independence: Theory and Evidence.» The MIT Press (1992).

[6] Bernanke, Ben S. et Ilian Mihov. «Measuring Monetary Policy.» The Quarterly Journal of Economics (Aug, 1998).

[7] Blancheton, Bertrand. «Central bank independence in a historical perspective. Myth, lessons and a new model.» Economic Modelling (2016): 101-107.

[8] Chappert, Magali. «Le Projet français de banque d'État du Maroc, 1889-1906.» Revue française d'histoire d'outremer (1975).

[9] Cukierman, Alex. «Central Bank Independence, Political Influence and Macroeconomic Performance: A Survey of Recent Developments.» Springer-Verlag Berlin · Heidelberg (1997).

[10] «Central Bank Strategy, Credibility, and Independence: Theory and Evidence.» The American Political Science Review, Vol. 87, No. 2 (1993): 519-520.

[11] Feiertag, Olivier. «L'organisation de Bank Al-Maghrib de 1959 à nos jours : l'émergence de la banque centrale au Maroc.» revue-histoire-economie-et-societe (2016).

[12] Mourougane, Annabelle. «Indépendance de la Banque centrale et politique monétaire: application à la Banque centrale euopéenne.» Revue française d'économie volume 13, n¹, (1998): 135-197.

[13] Le nouveau statut de BAM Objet de la loi n 76-03 entrée en vigueur le 20 Février 2006 